\title{
Implicit Upwind Methods for the Compressible Navier-Stokes Equations
}

T. J. Coakley

May 1983

\author{
LIBRARY COPY \\ JUN $6 \quad 1983$ \\ LANGLEY RESEARCH CENTER \\ LIBRARY, NASA \\ HAMPTON, VIRGINIA
}


NASA Technical Memorandum 84364

\section{Implicit Upwind Methods for the Compressible Navier-Stokes Equations}

T. J. Coakley, Ames Research Center, Moffett Field, California

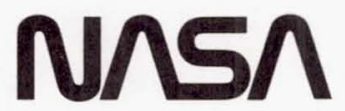

National Aeronautics and

Space Administration 


\section{Abstract}

A class of implicit upwind-differencing methods for the compressible Navier-Stokes equations is described and applied. The methods are based on the use of local eigenvalues or wave speeds to control spatial differencing of inviscid terms and are aimed at increasing the level of accuracy and stability achievable in computation. Techniques for accelerating the rate of convergence to a steady-state solution are also used. Applications to inviscid and viscous transonic flows are discussed and compared with other methods and experimental measurements. It is shown that accurate and efficient transonic airfoil calculations can be made on the Cray-1 computer in less than 2 min.

\section{Introduction}

The purpose of this paper is to describe a class of implicit upwind-differencing methods (IUM) for the compressible Euler and Navier-Stokes equations. The methods use local eigenvalues or wave speeds to control inviscid spatial differencing and are closely related to many other recent techniques. ${ }^{1-9}$ The objective in all these methods is to achieve more stable and accurate solutions than can be obtained using conventional techniques such as the central-differencing method 10 and MacCormack's method. ${ }^{11}$ In the latter two methods, dissipative terms must usually be added to control parasitic oscillations, and the choice of the form of the dissipation terms and the size of the dissipation constants is difficult. The more recent methods, utflizing local eigenvalues, are naturally dissipative and, in principal, do not require the addition of extra terms to stabilize calculations. In practice, however, these methods also have difficulties, especially in regions of the flow where the eigenvalues change sign, and again special procedures must frequently be introduced to improve accuracy. $1-3$

The class of mechods reported in this paper is an extension of the second-order method reported in Ref. I where dissipative terms scaled on the squares of the local eigenvalues were used. Although the method of Ref. 1 is simpler than the present methods, it was found in subsequent appl1cations to suffer the same difficulty as that of earlier methods, that is, the need for fine tuning of dissipation constants to achieve accurate solutions. Of the class of methods to be reported in this paper, one second-order upwind method (UW2II) has been found, through extensive numerical experimentation, to produce accurate solutions of transonic-flow problems without the need for special treatment at normal shock waves or sonic lines. For oblique shock waves, the method produces small undershoots or overshoots around the shock which may be substantially reduced by a simple rechnique. The other methods reported produce accurate

*Research Scientist. Member AIAA.

This paper is declared a work of the U.S. Government and therefore is in the public domain. solutions, except at normal shock waves, where they may be improved by special treatments.

The methods presented are closely related to the flux-vector splitting method of Steger and Warming, ${ }^{3}$ who used the finite-difference technique. The present methods are designed for incorporation into the strongly conservative finite-volume technique ${ }^{1}$ which, in contrast to the former technique, retains the property of free-stream maintenance in curvilinear coordinates without the need for differencing metrics in the same way as fluxes.

In this paper, a one-dimensional description of the methods will be made followed by a discussion of two-dimensional results which include inviscid and viscous transonic-flow calculations. These are compared with other numerical methods and experimental results. A basic conclusion from these results is that accurate and efficient transonic airfoil calculations can be made, using the full Navier-stokes equations, in less than $2 \mathrm{~min}$ on the Cray-1 computer.

\section{Theory}

Although the methods will be applied to twodimensional viscous-flow problems in curvilinear coordinates, they will be described here in the inviscid, one-dimensional context in order to simplify the development. The one-dimensional Euler equations may be written

$$
\partial_{t} U+\partial_{x} F=0
$$

where $\partial_{t}=\partial / \partial t, \partial_{x}=\partial / \partial x$, and

$$
U=(\rho, \rho u, \rho E)^{T}, \quad E=\left(\rho u, \rho u^{2}+p,(\rho E+p) u\right)^{T}
$$

are the conservative state and flux vectors with $\rho=$ density, $u=$ velocity, $p=\rho c^{2} / Y=p r e s s u r e$, $O E=p /(y-1)+\rho u^{2} / 2=$ total energy, and $c=$ sound speed. The aonconservative state vector $V$ and the Jacobian of the flux vector $A$ are written

$$
V=(\rho, u, p)^{T}, \quad A=\partial F / \partial U=R^{-1} \Lambda R
$$

where $\Lambda=\operatorname{dlag}(u, u+c, u-c)$ is the diagonal matrix of eigenvalues of $A$, and $R$ is a similarity matrix diagonalizing $A$. The matrix $R$ may be written

$$
\begin{aligned}
& R=Q P, \quad P=\partial V / \partial U \\
& Q=\left[\begin{array}{ccc}
-c^{2} & 0 & 1 \\
0 & \rho c & 1 \\
0 & -\rho c & 1
\end{array}\right] \quad P=\left[\begin{array}{ccc}
1 & 0 & 0 \\
-u / 0 & 1 / \rho & 0 \\
\frac{X}{2} u^{2} & -X u & x
\end{array}\right]
\end{aligned}
$$

where $X=\gamma-1$ and $Y$ is the ratio of specific heats. 
The sign of $A$ and the absolute value of $A$ are defined by

$$
\begin{aligned}
S & =\operatorname{sgn} A=R^{-1} \operatorname{sgn} \Lambda R \\
|A| & =a b s A=R^{-1} \text { abs } \Lambda R=S A=A S
\end{aligned}
$$

where sgn $\Lambda$ and abs $\Lambda$ are diagonal matrices consisting of the signs and the absolute values of the eigenvalues, respectively.

The basic implicit algorithm, using firstorder time-differencing, may be written in the delta form (leaving spatial differencing arbitrary) as

$$
\left(I+\Delta t \partial_{x} A\right) \Delta U=-\Delta t \partial_{x} F
$$

where $\Delta U=U(x, t+\Delta t)-U(x, t)$ is the delta variable and $\Delta t$ is the time-step.

In order to completely define the algorithm, the spatial derivatives in Eq. (6) must be replaced by spatial difference operators. We consider first the spatial flux differencing, or the right-handside term of Eq. (6). It is expressed in the following generic (finite-volume) form

$$
\partial_{x} F=\left(\hat{F}_{1+1 / 2}-\hat{F}_{1-1 / 2}\right) / \Delta x
$$

where $\Delta \mathrm{x}$ is the mesh spacing and $\hat{\mathrm{F}}_{1+1 / 2}$ is the flux vector defined at the midpoint (or cell face) between the mesh points 1 and $1+1$. The state vector, $U(x, t) \rightarrow U(i \Delta x, a \wedge t)=U_{1}^{n}$, is defined at the mesh points, and the flux vector, $\hat{F}_{1+1 / 2}$, is defined in terms of $U_{1-1}, U_{1}, U_{1+1}, U_{1+2}$ as follows:

$$
\hat{\mathrm{F}}_{1+1 / 2}=1 / 2\left(F_{1}+F_{1+1}-D_{1+1 / 2}\right)
$$

where $F_{i}=F\left(U_{1}\right)$ and $D_{1+1 / 2}$ is a dissipation function to be specified. If $D_{1+1 / 2}=0$, the scheme reduces to pure central-differencing. We note that the differencing of Eq. (7) is fully conservative, regardless of the definition of $D_{1+1 / 2}$, since it represents a telescoping sum of terms.

The dissipation function, $D_{1+1 / 2} \rightarrow D$, 1n Eq. (8) will be expressed in terms of sparialdifference operators, which are defined as follows. We let $W$ be either $F, U$, or $V$, and we define the operators, $\delta_{\ell}, N_{\ell}, N_{l}$, by

$$
\begin{aligned}
& \delta_{\ell} W_{i+1 / 2}=T_{i+1 / 2}^{\ell}\left(W_{1+1}-W_{1}\right) \rightarrow \delta_{\ell} W \\
& \mathscr{M}_{2} W_{1+1 / 2}=\alpha\left(\delta_{\ell} W_{1+3 / 2}-\delta_{\ell} W_{1-1 / 2}\right) \rightarrow \omega_{\ell} W \\
& \boldsymbol{r}_{2} W_{1+1 / 2}=\beta \delta_{\ell} W_{1+1 / 2}-\gamma\left(\delta_{2} W_{1+3 / 2}+\delta_{\ell} W_{1-1 / 2}\right) \\
& -w_{2} \mathrm{~W}
\end{aligned}
$$

The constants $\alpha, \beta$, and $\gamma$ define the spatial accuracy of the differencing and will be discussed later. The subscript $\&$ takes the values 1,2 , and 3 , and the matrices $T_{i+1 / 2}^{\ell} \rightarrow T^{l}$ are defined

$$
T^{l}=I, T^{2}=R, T^{3}=Q
$$

A suitable average must be given for $T^{\ell}$ in terms of $U_{1}, U_{1+1}$ for $\ell=2,3$, and this averaging will be discussed below.
The present methods may be developed in a sequence of steps starting with the flux-vector splitting method of Steger and Warming. ${ }^{3}$ The latter method can be expressed in terms of Eqs. (7) and ( 8$)$, using the dissipation function

$$
D=N_{1} \mathrm{AU}+N_{1}|\mathrm{~A}| \mathrm{U}
$$

and assuming that the flux vectors $\left(F_{1}\right.$ and $F_{i+1}$ ) in Eq. (8) possess the homogeneous property, that is, $\mathrm{F}=\mathrm{AU}$. However, it is not necessary to represent the flux vectors in this manner and they may be used directly. Furthermore, $\mathrm{AU}$ and $|\mathrm{A}| \mathrm{U}$ in Eq. (11) may be replaced, using Eq. (5), by $F$ and SF so that Eq. (11) can be expressed alternatively by

$$
D=\mu_{1} F+\mu_{1} S F
$$

This results in a flux-vector splitting method that does not depend on the homogeneous property.

Unfortunately, the occurrence of the matrix $S$ inside the operator $\mathscr{N}_{1}$ causes difficulties in the flux-vector splitting method when the eigenvalues change sign. ${ }^{3}$ In this case the differencing becomes inconsistent, and special procedures must be used to stabilize the calculations. ${ }^{2}$

An alternative form of the method, here called Mechod I, was proposed by Hwang ${ }^{6}$ (in one-dimensional first-order form for the pseudounsteady Euler equations). This form is obtained from Eq. (12) by taking the matrix $S$ outside the operator, that is,

$$
\text { (Method I) } \quad D=M_{1} F+S K_{1} F
$$

where a sultable average must be defined for $S$. This method has the advantage that the differencing is more consistent when the eigenvalues change sign; numerical experiments in one-dimensica by Huang and in two-dimensions by the present athor using the first-order form and simple (Iinear) averaging produced good, normal shock captures. However, the second- and third-order forms, which are needed for accuracy in multidimensions, were found (by the present auchor) to be weakly unstable at normal shock waves, and spectal procedures were required to remove oscillations. The higher-order methods were also tried, using nonlinear averaging, and with the same kind of result. This method, although the most elegant of the three methods to be discussed, was therefore put aside in favor of the second method, now to be discussed.

Method II may be obtained from Eqs. (9) and (13) by the replacement

$$
\delta_{1} F \rightarrow A \delta_{1} U=A_{1+1 / 2} \delta_{1} U_{1+1 / 2}
$$

The operators $M_{1} F$ and $S n_{1} F$ are then replaced as follows :

$$
\left.\begin{array}{l}
N_{1} F \rightarrow A N N_{1} U=R^{-1} \Lambda R N_{1} U \rightarrow R^{-1} \Lambda N_{2} U \\
S a K_{1} F+S A_{a} K_{1} U=R^{-1}|\Lambda| R N_{1} U \rightarrow R^{-1}|\Lambda| N_{2} U
\end{array}\right\}
$$

so that the dissipation function of Method II can be written

$$
\text { (Method II) } D=R^{-1}\left(\Lambda H_{2} U+|\Lambda| r_{2} U\right)
$$


or

$$
\begin{aligned}
D_{i+1 / 2}= & R_{i+1 / 2}^{-1}\left(\Lambda_{i+1 / 2} N_{2} \mathrm{~J}\right. \\
& \left.+|\Lambda|_{i+1 / 2}{ }_{2} U_{i+1 / 2}\right)
\end{aligned}
$$

Method II has been found to produce clean and accurate (normal) shock captures in numerical experiments and is the basis of the results to be reported.

Method III is closely related to Method II but uses the nonconservative state vector, $V$. It is obtained through the replacements

$$
\delta_{1} \mathrm{U} \rightarrow \mathrm{P}^{-1} \delta_{1} \mathrm{~V}, \quad \boldsymbol{N}_{1} \mathrm{U} \rightarrow \mathrm{P}^{-1} \boldsymbol{\mu}_{1} \mathrm{~V}, \quad \boldsymbol{N}_{1} \mathrm{U} \rightarrow \mathrm{P}^{-1} \boldsymbol{N}_{1} \mathrm{~V}
$$

in Eq. (15). By noting that $R P^{-1}=Q$, the replacements in Eq. (15) can alternatively be taken as

$$
\begin{aligned}
& \mathscr{N}_{1} \mathrm{~F} \rightarrow \mathrm{A} \mathscr{N}_{1} U \rightarrow \mathrm{R}^{-1} \Lambda \mathrm{Q} \mathscr{N}_{1} \mathrm{~V} \rightarrow \mathrm{R}^{-1} \Lambda \mathscr{N}_{3} V \\
& S \mathscr{N}_{1} \mathrm{E}-\mathrm{SA} \mathscr{H}_{1} \mathrm{U} \rightarrow \mathrm{R}^{-1}|\Lambda| \mathrm{Q} \mathscr{N}_{1} V \rightarrow \mathrm{R}^{-1}|\Lambda| \mathscr{N}_{3} V
\end{aligned}
$$

so that the dissipation function of Method III can be written

$$
\text { (Method III) } \quad D=R^{-1}\left(\Lambda \mathbb{N}_{3} V+|\Lambda| \mathbb{N}_{3} V\right)
$$

Only limited numerical experimentation has been done with this method, and no results will be shown for it.

It is important to note that the replacements in Eqs. (15) and (18), indicated by the arrows, are equalities only if $A$ is constant; as a result, the methods may be expected to produce different results, especially at singular points where the eigenvalues change sign. As a further note, we may add that the form of the dissipation functions of Methods II and III was inspired by a study of Harten's explicit method 5,7 so that a more complete understanding of the present method, and perhaps even further improvements (e.g., entropy cond1tions) may be achieved by a careful study of his and related works.

In order to completely specify the algorithms, the method of averaging, that is, compuring $S_{1+1 / 2}$, $R_{i+1 / 2}$, etc., in terms of $U_{1}$ and $U_{1+1}$ or $V_{1}$ and $V_{i+1}$, must be given. Although there are many possibilities, we have tried only two methods; linear averaging using the nonconservative state vector $\left(\right.$ e.g., $u_{i+1 / 2}=\left(u_{1}+u_{1+1}\right) / 2$ and $c_{1+1 / 2}=\left(c_{1}+c_{i+1}\right) / 2$ ) and nonlinear averaging after Roe $^{9}$ as implemented by Yee et al. ${ }^{7}$ In the latter case, the replacement $\delta_{1} F \rightarrow A \delta_{1} U$ becomes an exact equality and expresses Roe's property $U$. In the results to be discussed, Roe's averaging has been used. The specific formulas used in computing $R_{1+1 / 2}$ and $\Lambda_{1+1 / 2}$ for Method II are given below.

$$
\begin{aligned}
a & =\left(o_{i+1} / D_{i}\right)^{1 / 2} \\
u_{i+1 / 2} & =\left(a_{i+1}+u_{i}\right) /(1+a) \\
c_{i+1 / 2}^{2} & =(\gamma-1)\left[\left(a_{i+1}+H_{i}\right) /(1+a)-u_{i+1 / 2}^{2}\right] \\
H & =E+p / D
\end{aligned}
$$

These equations are used in computing $R_{i+1 / 2}$ from

\begin{tabular}{|c|c|c|c|c|c|c|c|c|}
\hline \multirow{2}{*}{ Scheme } & \multirow{2}{*}{$\begin{array}{l}\text { Desig- } \\
\text { nator }\end{array}$} & \multicolumn{3}{|c|}{ Coefficients } & \multirow{2}{*}{$\frac{\text { Nodal }}{1-1}$} & \multicolumn{2}{|c|}{ cluster, } & \multirow{2}{*}{$\frac{\hat{F}_{i+1 / 2}}{1+2}$} \\
\hline & & $\bar{a}$ & $B$ & $\bar{\gamma}$ & & 1 & $1+1$ & \\
\hline $\begin{array}{l}\text { Firse- } \\
\text { order } \\
\text { upwind }\end{array}$ & UW1 & 0 & 1 & 0 & - & $\odot$ & - & - \\
\hline $\begin{array}{l}\text { Second- } \\
\text { order } \\
\text { upwind }\end{array}$ & UW2 & $1 / 2$ & 1 & $1 / 2$ & $\odot$ & $\odot$ & $\cdot$ & - \\
\hline $\begin{array}{l}\text { Third- } \\
\text { order } \\
\text { upwind }\end{array}$ & UW3 & $1 / 6$ & $1 / 3$ & $1 / 6$ & $\odot$ & $\odot$ & $\odot$ & - \\
\hline $\begin{array}{l}\text { Second- } \\
\text { order } \\
\text { dissi- } \\
\text { pative }\end{array}$ & $\mathrm{CD} 2$ & 0 & $\varepsilon$ & $\varepsilon / 2$ & $\odot$ & $\odot$ & $\odot$ & $\odot$ \\
\hline
\end{tabular}
Eq. (4) where it may be noted that in the product $R=Q P$, the density cancels so that an average for $\rho$ is not required.

As stated earlier, the coefficients, $\alpha, \beta$, and $\gamma$, appearing in the dissipation operators $\boldsymbol{t}$ and * define the spatial accuracy of the flux differencing. Several options for these coefficients are listed in Table 1.

Table 1 Scheme options and nodal clusters

The nodal clusters are indicated for the midpoint flux vector, $\hat{\mathrm{F}}_{i+1 / 2}$ where $1 \mathrm{t}$ is assumed that all the eigenvalues of $A_{1+1 / 2}$, are positive. For the second-order dissipative scheme, that is, $\mathrm{CD} 2, \varepsilon$ is a free parameter. Assuming $A=$ constant, spatial accuracy may be checked by Taylor series expansion of Eq. (7) utilizing Eqs. (8), (9), and (13). The above schemes may be used with any of the three basic methods, which will be identified by placing a Roman numeral after the scheme designator. For example, the second order upwind method II w11l be denoted by UW2II.

As mentioned in the Introduction, the capture of oblique shock waves is degraded to some extent by the appearance of oscillations. These may be reduced substantially by a high resolution technique in which a switch to first-order, that is, forcing, $a, y \rightarrow 0$ and $B \rightarrow 1$, at points of local maxima or minima in the pressure. For the secondorder upwind method this is achieved through the following formulas:

$$
\begin{aligned}
\alpha & =\gamma=\max \left[0.5,0.5-\left(\delta^{\prime} p-\delta^{\prime \prime} p\right) / \varepsilon^{\prime}\right] \\
\delta^{\prime} p & =\left|\delta p_{1-1 / 2}\right|+\left|\delta p_{1+1 / 2}\right|+\left|\delta p_{1+3 / 2}\right| \\
\delta^{\prime \prime} p & =\left|\delta p_{1-1 / 2}+\delta p_{1+1 / 2}+\delta p_{1+3 / 2}\right| \\
\delta p_{1+1 / 2} & =p_{1+1}-p_{1}
\end{aligned}
$$

The parameter $\varepsilon^{\prime}$ is a small constant $\left(\varepsilon^{\prime} \approx(0.01-0.001) p_{\infty}\right)$ which controls the rate of approach to the first-order relations at those points where the pressure departs from monotonicity, that is, $\delta^{\prime} p>\delta^{\prime \prime} p$. The designator HR will be appended to the basic designator when this option is used, for example, UWIIHR. 
The spatial differencing of the implicit or left-hand-side terms of Eq. (6) follows the development given in Ref. 1. First-order upwind differencing in an (approximate) nonconservative scalar tridiagonal form is used. The algorithm may be written

$$
R^{-1}\left[I+\Delta t\left(\Lambda^{+} \nabla_{X}+\Lambda^{-} \Delta_{X}\right)\right] R \Delta U=-\Delta t a_{X} F
$$

where

$$
\begin{aligned}
\Lambda^{ \pm} & =(\Lambda \pm|\Lambda|) / 2 \\
\nabla_{x} W & =\left(W_{i}-W_{1-1}\right) / \Delta x \\
\Delta_{x} W & =\left(W_{i+1}-W_{i}\right) / \Delta x
\end{aligned}
$$

Advantages of this formulation are 1) it substantially reduces computing time compared with the more exact block-tridiagonal form, 2) the upwind differencing is dissipative which, coupled with the upwind dissipative differencing of the righthand side, enhances the (1inear) stability of the overall algorithm, and 3 ) viscous terms may be included by means of a simple technique. The main limitation of the formulation is that time accuracy is lost to some extent, but this is of no consequence in steady-state problems, which are the principal aim of this paper.

The inclusion of viscous terms is described as follows. The one-dimensional Navier-Stokes equations may be written

$$
\begin{gathered}
B=0^{-1}\left[\begin{array}{ccr}
\partial_{t^{U}}+\partial_{x}\left(F-B \partial_{x} U\right)=0 \\
0 & 0 & 0 \\
-\mu^{\prime} u & \mu^{\prime} & 0 \\
\left.\mu^{\prime \prime}-\mu^{\prime}\right) u^{2}-\mu^{\prime \prime} e & \left(\mu^{\prime}-\mu^{\prime \prime}\right) u & \mu^{\prime \prime}
\end{array}\right] \\
\mu^{\prime}=(4 / 3) \mu, \quad \mu^{\prime \prime}=(\gamma / \operatorname{Pr}) \mu, \quad \operatorname{Pr}=C_{P} \mu / \mathrm{K}
\end{gathered}
$$

where $u$ is the molecular viscosity, Pr is the Prandt 1 number, and $e$ is the specific internal energy, $e=c^{2} / y(y-1)$. The viscous analog of the time-differenced delta-form algorithm, that is, Eq. (6), becomes

$$
\left[I+\Delta t \partial_{x}\left(A-B \partial_{x}\right)\right] \Delta U=-\Delta t \partial_{x}\left(F-B \partial_{x} U\right)
$$

where on the left-hand side of this equation the matrix B, like A, is evaluated at the time-level $n$. The approximate scalar-tridiagonal form of this equation is derived by replacing $B$ by the matrix $V I$, where $I$ is the identity matrix, $v=\mu_{\max } / \rho$, and $\mu_{\max }$ is the largest eigenvalue of $B$, that is, $\mu_{\max }=\max \left(\mu^{\prime}, \mu^{\prime \prime}\right)$. In this case, the above equation, expressed in nonconservative form, becomes

$R^{-1}\left[I+\Delta t\left(\Lambda \partial_{x}-v \partial_{x}^{2}\right)\right] R \Delta U=-\Delta t \partial_{x}\left(F-B \partial_{x} U\right)$

Utilizing upwind differencing for the inviscid term: $\Lambda \partial_{x}$, and central differencing for the viscous term $v \partial_{x}^{2}$, the diagonal difference operator in this expression can be represented by

$$
\left\{I+(\Delta t / 2)\left\{\Lambda\left(\nabla_{x}+\Delta_{x}\right)-(|\Lambda| \Delta x+2 v) \Delta_{x} \nabla_{x}\right]\right\}
$$

where $\nabla_{x}$ and $\Delta_{x}$ are defined by Eq. (21). Conventlonal second-order central differencing is used for the viscous-flux differencing on the right-hand side of Eq. (24).

The numerical method, as outlined in the preceding paragraphs, may be generalized to multidimensional curvilinear coordinates utilizing the finite-volume technique, following the development of Ref. 1. Boundary conditions based on the method of characteristics are also described in Ref. 1 .

Spatially varying time-steps are used to accelerate 1terative convergence to a steady-state solution. The procedure used here is a modification of that described in Ref. 1. Expressed in terms of two-dimensional Cartesian coordinates, the relationship used for the local time-step is $\Delta t=C F L \cdot \min \left(\frac{\Delta x}{|u|+c}, \frac{\Delta y}{|v|+c}\right)$ $=\mathrm{CFL} \cdot \Delta t_{\text {local }}$

where CFL is the local Courant number, usually taken to be $O(5-10)$ for most inviscid problems. For viscous-flow problems at high Reynolds numbers, where a finely spaced mesh is used adjacent to solid surfaces, the above formula leads to very small values of $\Delta t$ in the neighborhood of the surface. In these cases, the formula is modified by placing a lower limit on $\Delta t$, that is,

$$
\Delta t=C F L \cdot \max \left(\Delta t_{10 c a l}, \Delta t_{0}\right)
$$

where $\Delta t_{0}$ is a constanc of order $O(\Delta x / c)$ when $y$ is the fine mesh direction.

\section{Results}

Representative calculations of invis id and viscous flows using the second-order methe, UW2II, are shown in F1gs. 1-9. These resucts are compared with those obtained from other methods and experiments.

Results for the inviscid transonic flow about an 187-thick, circular-arc airfoil are illustrated in Fig. 1, where surface-pressure distributions using the present method and method B2 of Ref. 1 are given. The two methods show good agreement except in the vicinity of the shock wave, where the present method shows a superior, three-point capture.

Computed surface pressures for the inviscid transonic flow about an NACA 0012 airfoll are shown in Fig. 2. This figure shows results obtained usin? the present method and those obtained from Ref. 2, using the central differencing and the (secondorder upwind) flux-splitting methods. The three methods are in basic agreement except in the neighborhood of the shock wave, where the present method shows a higher quality, two-point capture.

Figure 3 shows calculations of a shockreflection problem investigated by Yee et al. ${ }^{7}$ Figures $3 a$ and $3 b$ show contour plots and pressure distributions using the present method UW2II. Figures $3 c$ and $3 d$ show results using the present method with the high-resolution option added, UW2IIHR. (This is the only case in which the highresolution option was used). These results may be 
compared with the results of Ref. 12, which is contained in the present volume, and those of Ref. 7. It may be stated that the present method, with the high-resolution option off, gives essentially the same results as those obtained using the secondorder flux-splitting method. ${ }^{7}$ This is related to the fact that the two methods reduce to a single method in the linear constant-coefficient case. The high-resolution option of the present method gives results roughly comparable to those of Hartens method (explicit TVD) and to those of the implicit TVD method (FTVD) of Yee et al. 12 An advantage of the present implicit method and the implicit TVD method over the explicit TVD method is that the implicit and implicit TVD methods do not suffer an explicit time-step limitation and can be run at Courant numbers greater than 1 . The present method was run at a Courant number of 5 , and convergence was achieved in approximately 60 time-steps. This is comparable to the performance of the implicit TVD method.

Figure 4 compares calculations of a laminar supersonic flow over a flat plate at a low Reynolds number. In this case, a strong interaction at the place leading edge results in a shock wave and a nonuniform pressure distribution. The present method is compared with results from MacCormack's explicit-implicit method ${ }^{11}$ (those results were provided by J. Viegas, Ames Research Center). Although not shown, comparisons of surface-pressure and skin-friction distributions indicate that the two methods are in close agreement. F1gure $4 \mathrm{~b}$ shows pressure distributions normal to the plate, intersecting the shock wave. In this case, the shock resolution of MacCormack's method is degraded by comparison with the present method.

Figures 5-9 show results of transonic-airfoil calculations, at high Reynolds numbers, requiring the use of turbulence models. Flgure 5 shows the mesh system used. The mesh is analyotcally generated and consists of a $120 \times 50$ cell C-grid. A sheared conformal mapping is used over the front half of the airfoil, and an H-type mesh is used over the back half and in the wake. The mesh points are exponentially spaced away from the alrfoil, and the cell spacing adjacent to the surface corresponds to a $\mathrm{y}^{+}$of approximacely 0.8 . About 23 mesh points are contained in the boundary layer at the midpoint, and the outer boundary is placed at 10 chord lengths from the airfoil.

Boundary conditions used in the airfoil calculations were as follows: no-slip velocity conditions at the airfoll surface, constant total enthalpy and entropy (or constant total pressure) conditions around the outer " $C$ " part of the mesh boundary, and constant (free-stream) static pressure conditions along the vertical back boundary. Further details on these boundary conditions are given in Ref. 1.

Figure 6 shows results for an NACA 0012 airfoil at the same Mach number and angle of attack as used in the inviscid results (see Fig. 2). Figure 6a shows computed Mach contours, and F1g. 6b shows computed pressure distributions. These are compared with preliminary experimental results (from the Ames high Reynolds Number Channel II facility) obtained by J. McDevitt, L. Hand, and A. Okuno. The calculations for this case were done with the full Navier-Stokes equations, using the zero-equation turbulence model of Cebec 1 and
Smith, without pressure-gradient correction. In the experiment, the wind-tunnel walls were adjusted to conform to free-air streamlines so that interference effects should be minimized. The (freeair) calculations using the present method are in good agreement with experiment. Calculations of this flow using various two-equation turbulence models have also been made and are reported in a companion paper. 13

Figures 7 and 8 show results for the RAE 2822 airfoil 14 which was used as an experimental test case at the 1981 Stanford conference on Complex Turbulent Flows. 15 Figure 7 corresponds to the subcritical Case 1 of the experiment, and Fig. 8 corresponds to the supercritical Case 9. Computed Mach contours are shown in Figs. $7 a$ and $8 a$, and compured pressure distributions are compared with experimental distributions in Figs. $7 \mathrm{~b}$ and $8 \mathrm{~b}$. The calculations were done at the nominal test Mach number and the geometric angle of attack, and do not account for wind-tunnel interference effects. They were made using a new two-equation model which is reported in Ref. 13. A more derailed comparison of computational and experimental results is also included in Ref. 13.

Compurational efficiency of the present method is indicated in FIg. 9, which shows residual decay and lift convergence histories for the subcritical RAE airfoil calculation. The calculations used a spatially varying time-step in the inviscid flow ${ }^{1}$ and a constant time-step in the viscous boundary-layer flow; 3 min were required for 500 steps on the Cray-1 computer. Since lift convergence (and drag convergence, which is not shown) occurs at about 200 time-steps, the actual computing time required for practical applications in this case would be $1.2 \mathrm{~min}$. The computer code is partially vectorized (1.e., vector right-hand side and scalar left side) and a speed up by a factor of 2 may be expected if the complete code is vectorized. An additional speed up may be achieved by optimizing the local time-step, and work in this direction is currently in progress.

\section{Concluding Remarks}

We have described a class of implicit upwinddifferencing methods for solving the compressible Euler and Navier-Stokes equations. The methods are based on the use of local elgenvalues or wave propagation speeds to control inviscid spatial differencing and are aimed at achieving more accurate and stable solutions than can be obtained using more conventional methods. Of the class of methods investigated, one second-order implicit upwind method, UW2II, was found to produce accurate solutions of transonic-flow problems without the need for special treacments at normal shock waves or sonic lines. By combining the present method with acceleration techniques based on spatially varying time-steps, an effective and efficient algorithm is produced for solving compressible viscous-flow problems.

\section{References}

${ }^{1}$ Coakley, I. J., "Numerical Method for Gas Dynamics Combining Characteristic and Conservation Concep.rs," AIAA Paper 81-1257, June 1981. 
2Buning, P. G. and Steger, J. L., "Solution of the Two-Dimensional Euler Equations with Generalized Coordinate Transformation Using Flux Vector Splitting," AIAA Paper 82-0971, June 1982.

${ }^{3}$ Steger, J. L. and Warming, R. F., "Flux Vector Splitting of the Inviscid Gas Dynamics Equations with Application to Finite Difference Methods," Journal of Computational Physics, Vol. 40, No. 2, April 1982, p. 283.

${ }^{4}$ Chakravarthy, S. R. and Osher, S., "Numerical Experiments with the Osher Upwind Scheme for the Euler Equations," AIAA Paper 82-0975, June 1982.

${ }^{5}$ Harten, A., "A High Resolution Scheme for the Computation of Weak Solutions of Hyperbolic Conservation Laws," NYU Report, New York University, New York, N.Y., March 1982.

${ }^{6}$ Huang, L. C., "Pseudo-Unsteady Difference Schemes for One-Dimensional Fluid Dynamics Problems," Journal of Computarional Physics, Vol. 42, 1981, pp. 195-211.

${ }^{7}$ Yee, H. C., Warming, R. F., and Harten, A., "On the Application and Extension of Harten's High Resolution Scheme," NASA TM-84256, June 1982.

${ }^{8}$ Lombard, C. K., Oliger, J., and Yang, J. Y., "A Natural Conservative Flux Difference Splitting for the Hyperbolic Systems of Gas Dynamics," ALAA Paper 82-0976, June 1982.

${ }^{9}$ Roe, P. L., "Approximate Reimann Solvers, Parameter Vector, and Difference Schemes," Journal of Computational Physics, Vol. 43, 1981, pp. 357372 .

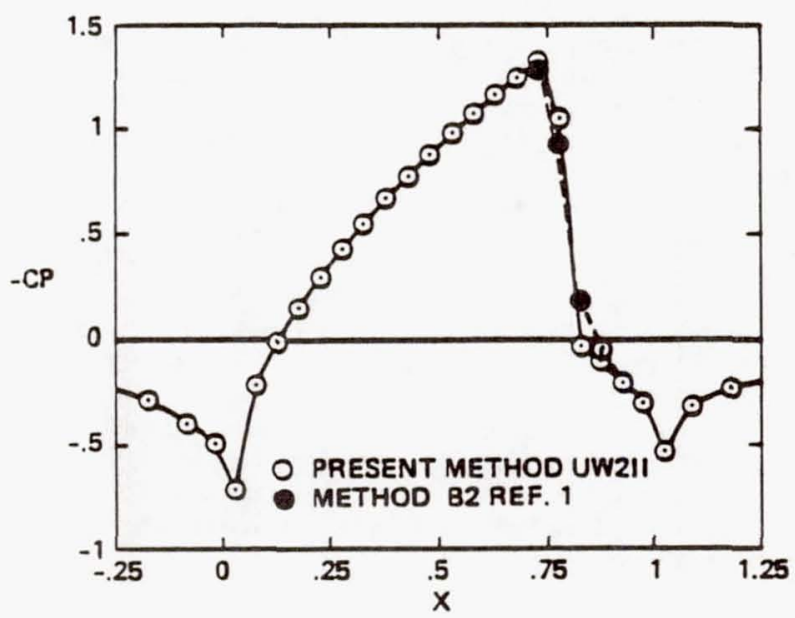

Fig. 1 Comparison of computed surface pressures for inviscid transonic flow about an $18 \%$-chick, circular-arc airfoil: $M=0.785,40 \times 20$ cell Hmesh.
${ }^{10}$ Beam, R. and Warming, R., "An Implicit Factored Scheme for the Compressible Navier-Stokes Equations," AIAA Journal, Vo1. 16, No. 4, 1978, pp. $393-402$.

${ }^{1}$ MacCormack, R. W., "A Numerical Method for Solving the Equations of Compressible Viscous Flow," AIAA Paper 81-0110, Jan. 1981.

${ }^{12}$ Yee, H. C., Warming, R. F., and Harten, A., "Implicit Total Variation Diminishing (TVD) Schemes for Steady-State Calculations," AIAA Paper 83-1902, July 1983.

${ }^{13}$ Coakley, T. J., "Turbulence Modeling Mechods for the Compressible Navier-Stokes Equations," AIAA Paper 83-1693, July 1983.

${ }^{14}$ Cook, P. H., McDonald, M. A., and Firmin, M. C. P., "AEROFOIL RAE 2822 - Pressure Distributions, and Boundary Layer and Wake Measurements," AGARD Advisory Report No. 138, 1979.

15 Kline, S. J., Cantwell, B. J., and Lilley, G. M., Session XI, Flow 8620, Transonic Airfoils, Proceedings of $1980-81$ AFOSR-HTTM-Stanford Conference on Complex Turbulent Flows, Vol. 1, 1981.

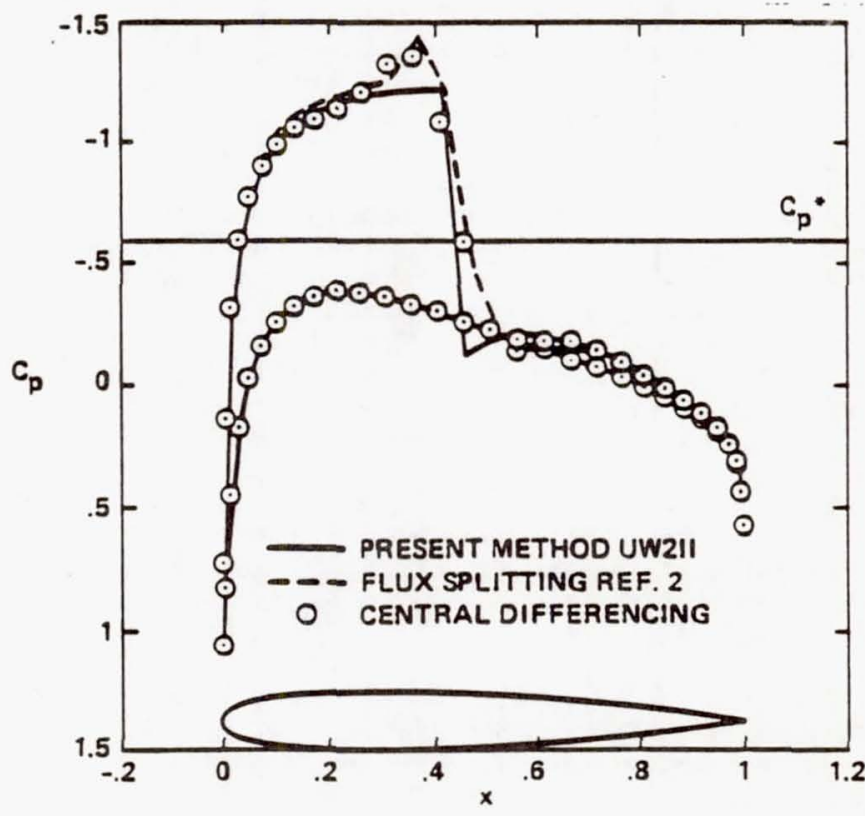

Fig. 2 Comparison of computed surface pressures for inviscid transonic flow about an NACA 0012 airfoil: $M=0.75, \alpha=2^{\circ}, 80 \times 20$ cell $C$-mesh $(79 \times 31$ mesh for Ref. 2). 

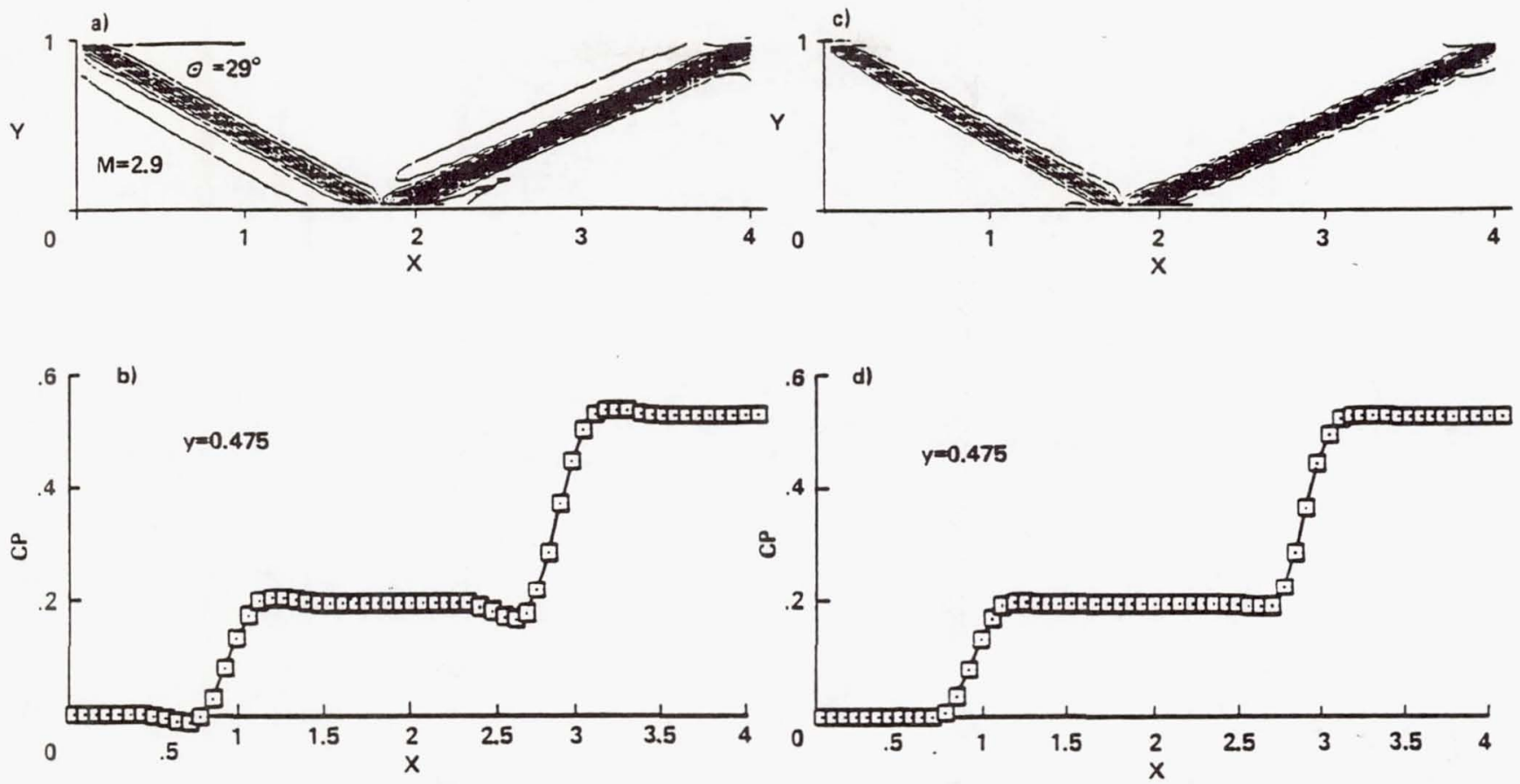

Fig. 3 Comparison of computed pressure contour levels and distributions for inviscid shock-reflection problem: $M=2.9, \theta=29^{\circ}, 60 \times 20$ cell H-mesh. a) Contour plots, present method UW2II; b) Pressure distributions, method UW2II; c) Contour plots, method UW2II-HR; d) Pressure d1stributions, method UW2II-HR.
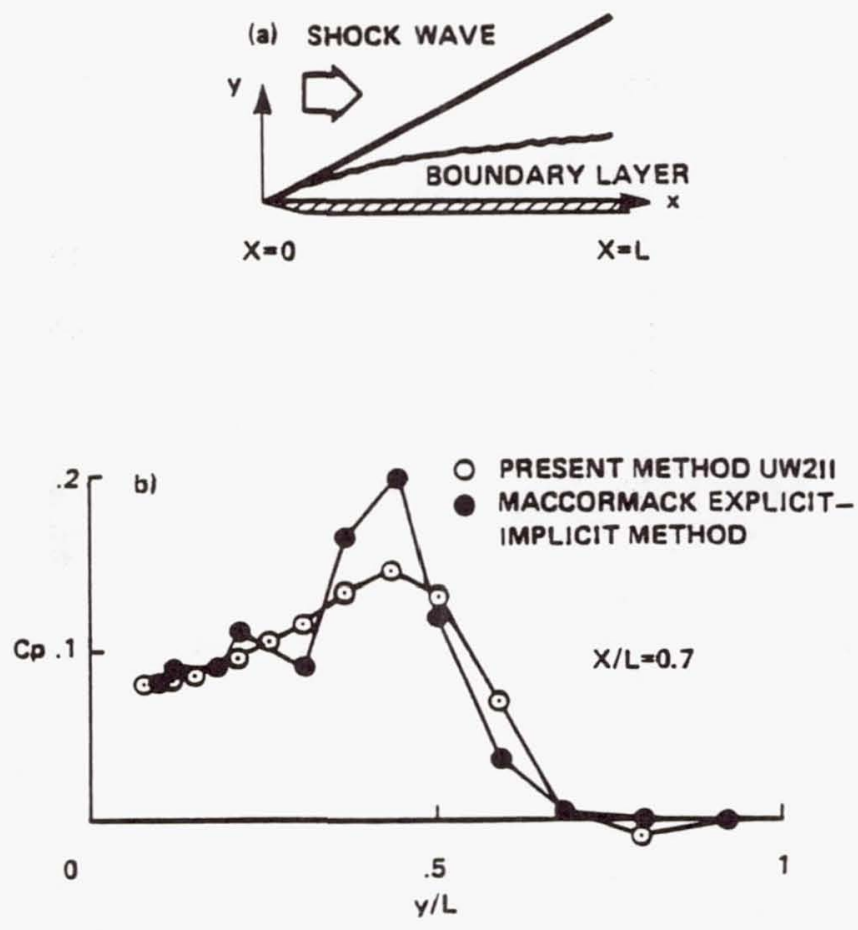

Fig. 4 Viscous supersonic leading-edge flow. a) Sketch of flow; b) Pressure distributions normal to plate surface. 


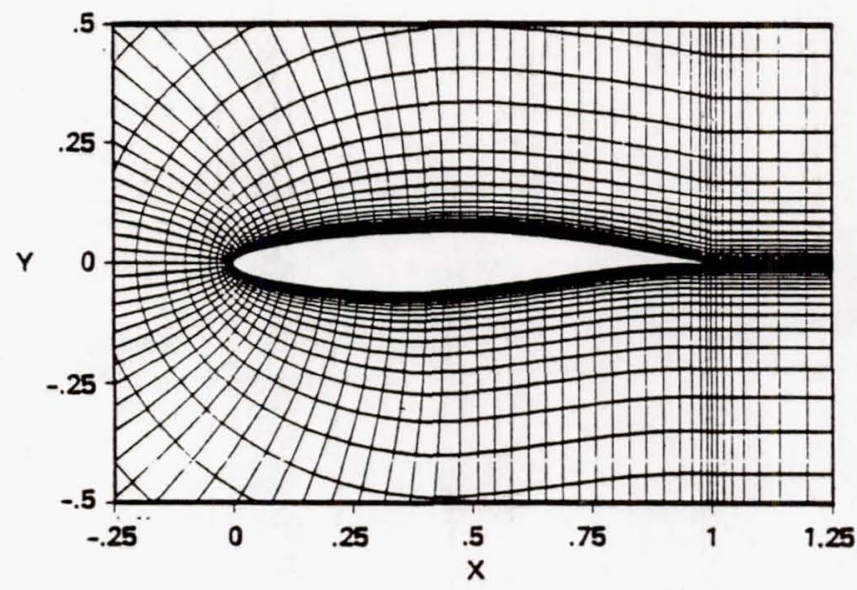

Fig. 5 Representative $(120 \times 50)$ C-mesh system used in viscous transonic airfoil calculations.

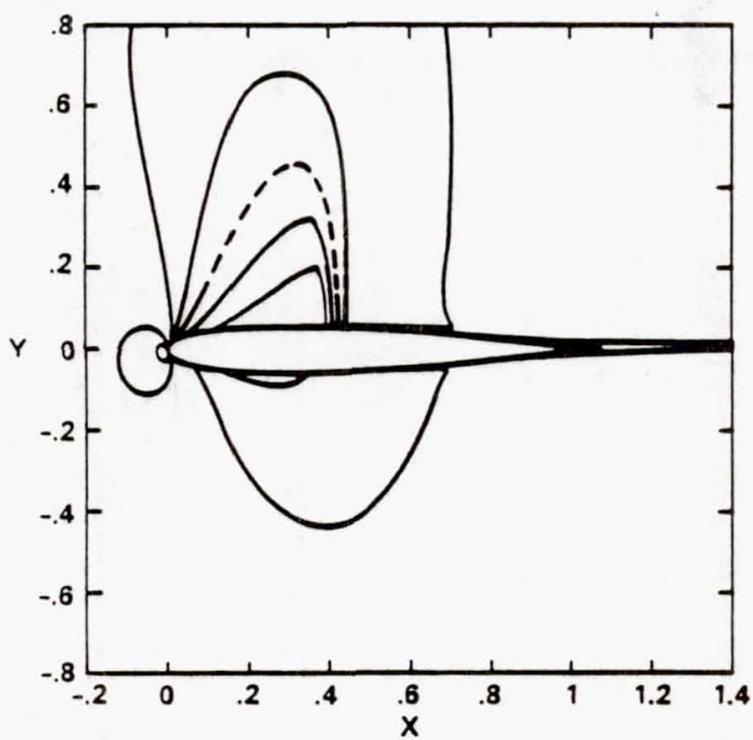

a) Computed Mach contours.

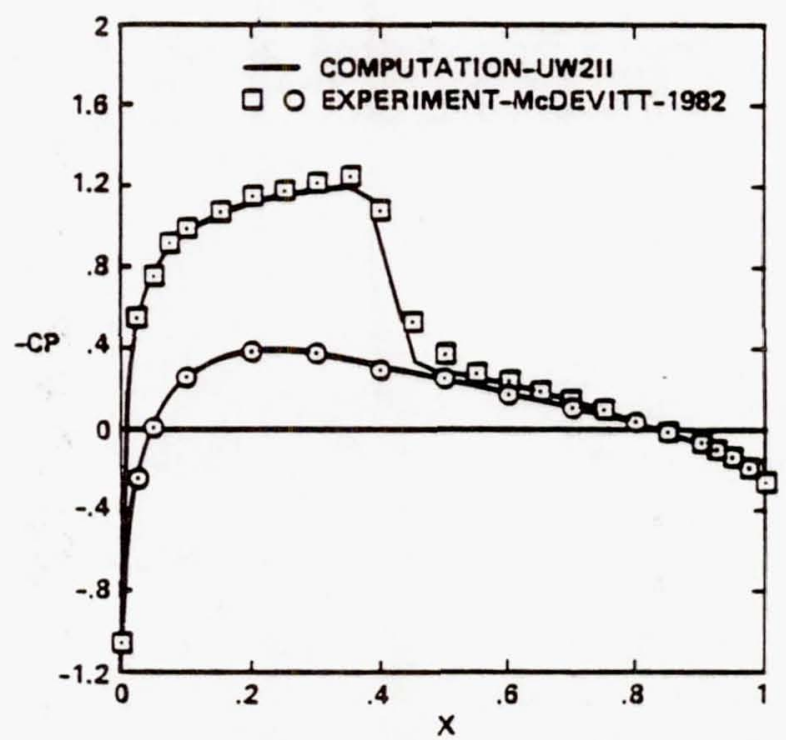

b) Experimental and computed surface pressures.

Fig. 6 Transonic flow about NACA 0012 airfoil: $M=0.75, \operatorname{Re}=10^{7}, a=2^{\circ}, 120 \times 50$ cell C-mesh, Cebeci-Smith 0-equation model. 


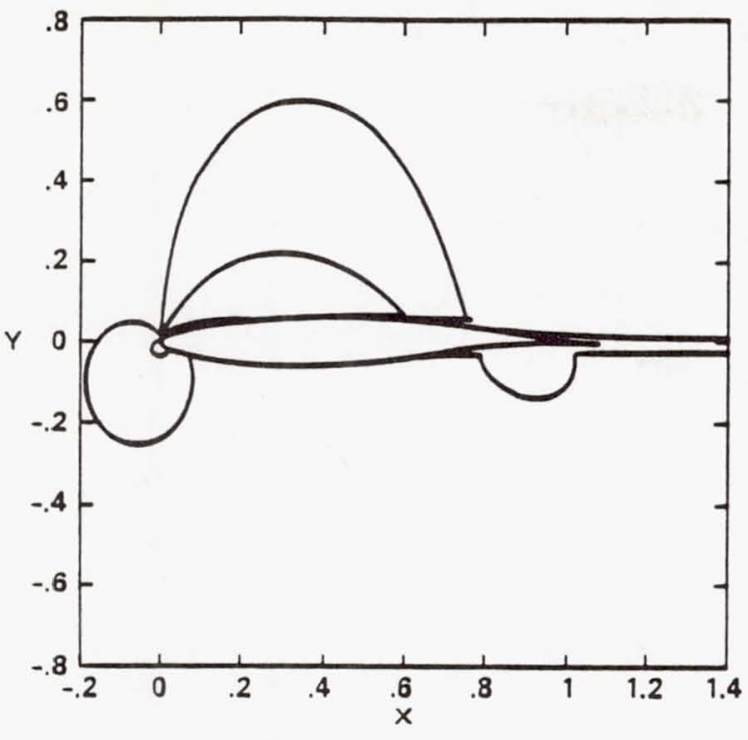

a) Compured Mach contours.

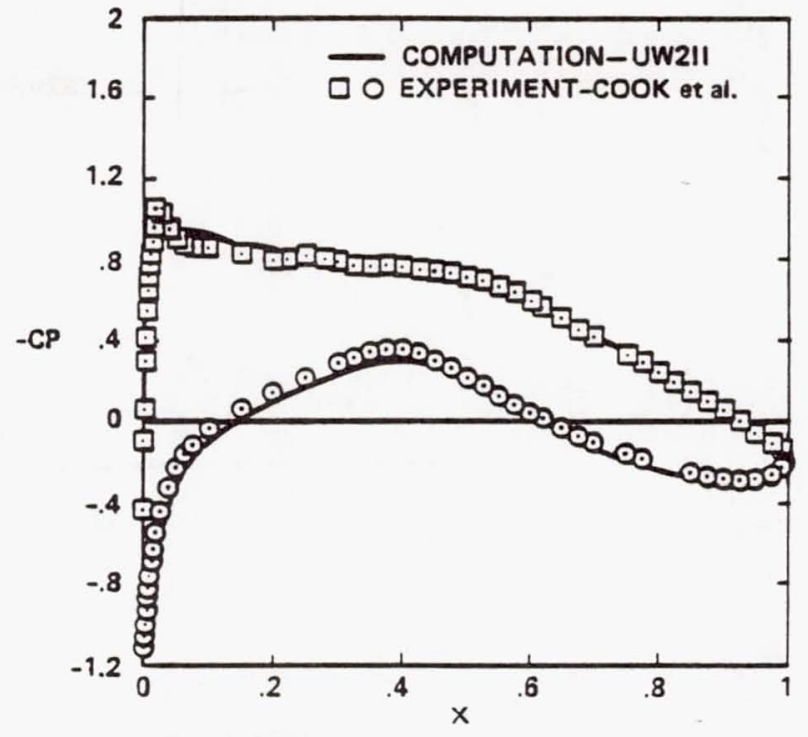

b) Experimental and computed surface pressures.

Fig. 7 Transonic flow about RAE 2822 airfoil, Case 1: $M=0.676$, Re $=5.7 \times 10^{6}, \alpha=2.4^{\circ}$, $120 \times 50$ cell C-mesh, q-w two-equation model.

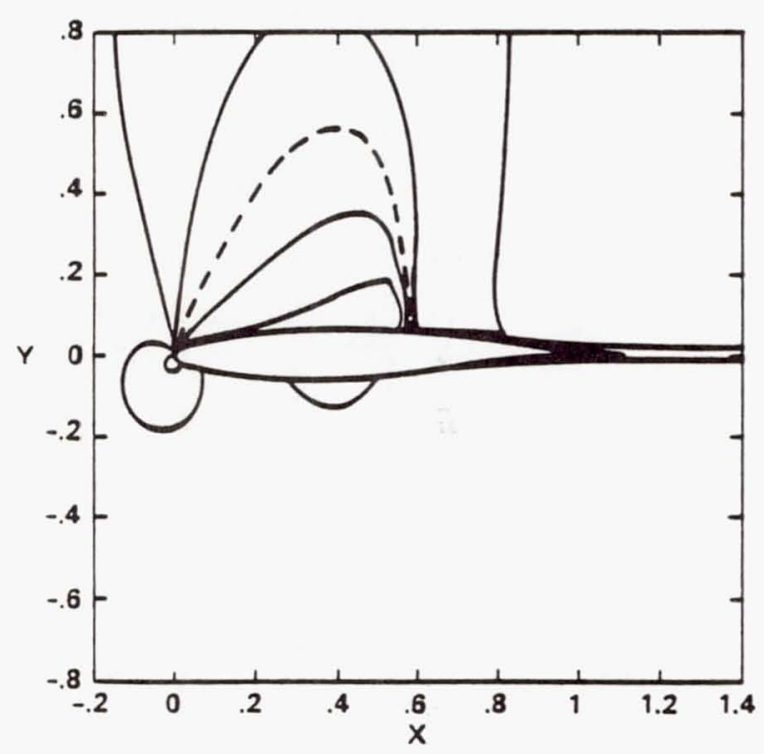

a) Computed Mach contours.

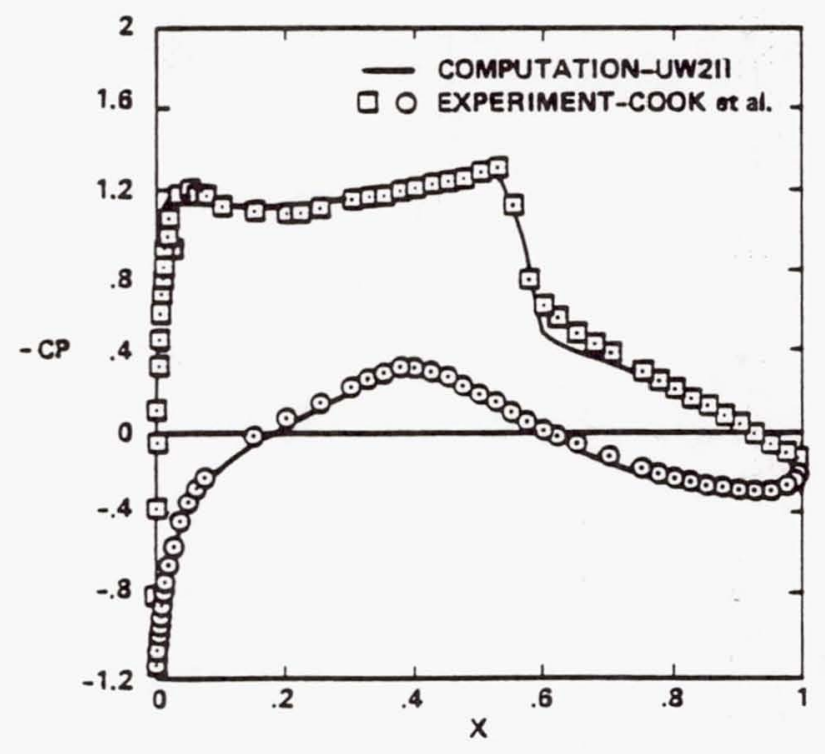

b) Experimental and computed surface pressures.

Fig. 8 Transonic flow about RAE 2822 airfoil, Case 9: $M=0.73, \operatorname{Re}=6.5 \times 10^{6}, \mathrm{CFL}=6, \mathrm{NE}=500$, $\mathrm{TCR}=0.12, \alpha=3.19,120 \times 50$ cell $\mathrm{C}$-mesh, $q-w$ two-equation model. 


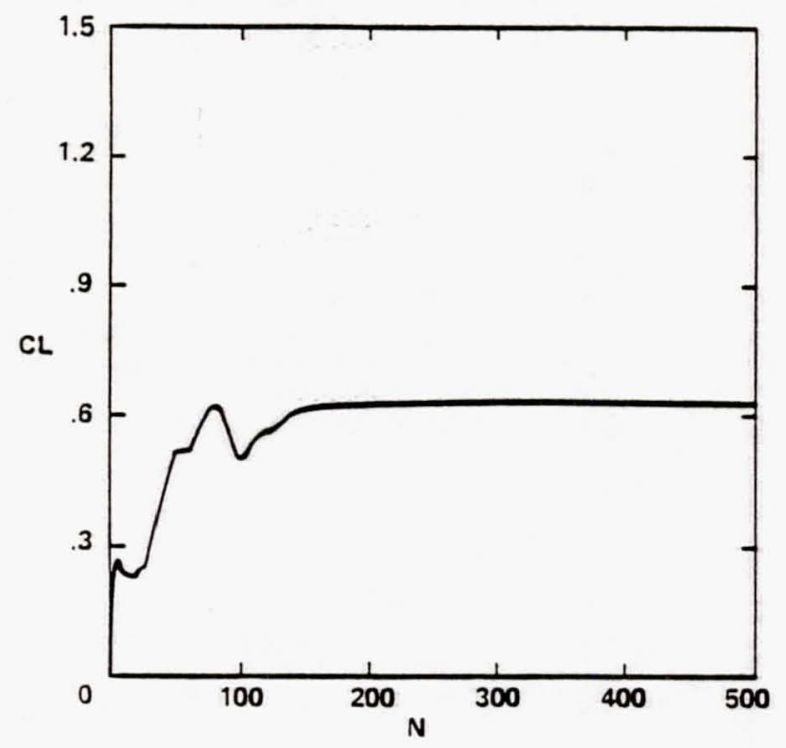

a) $\mathrm{L}_{2}$ residual history.

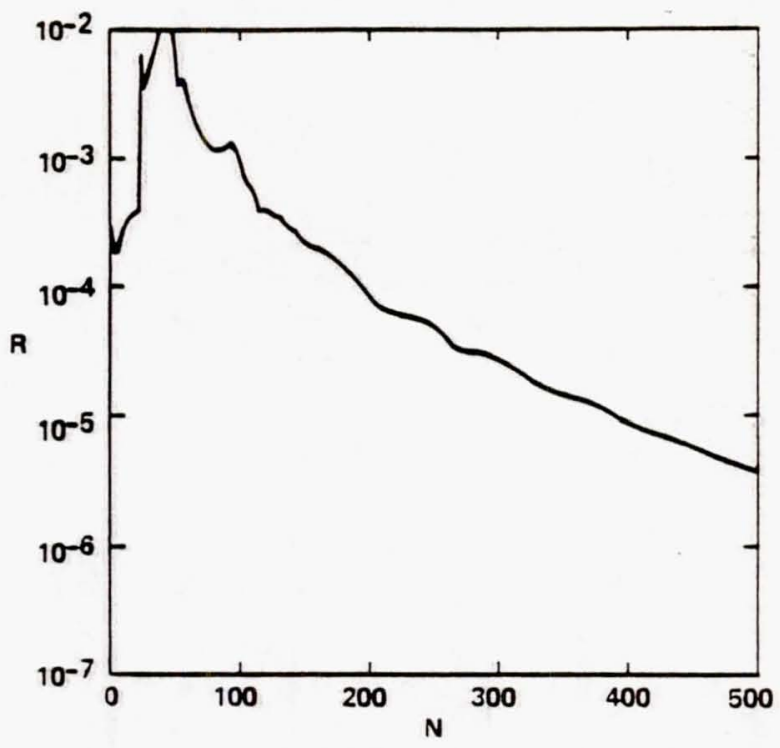

b) Lift history.

Fig. 9 Convergence histories for RAE 2822 airfoil computation, Case 1: $M=0.676, \operatorname{Re}=5.7 \times 10^{6}$, $\alpha=2.4^{\circ}, \mathrm{CFL}=8,120 \times 50 \mathrm{C}$-mesh, $\mathrm{q}-\mathrm{w}$ two-equation model. 


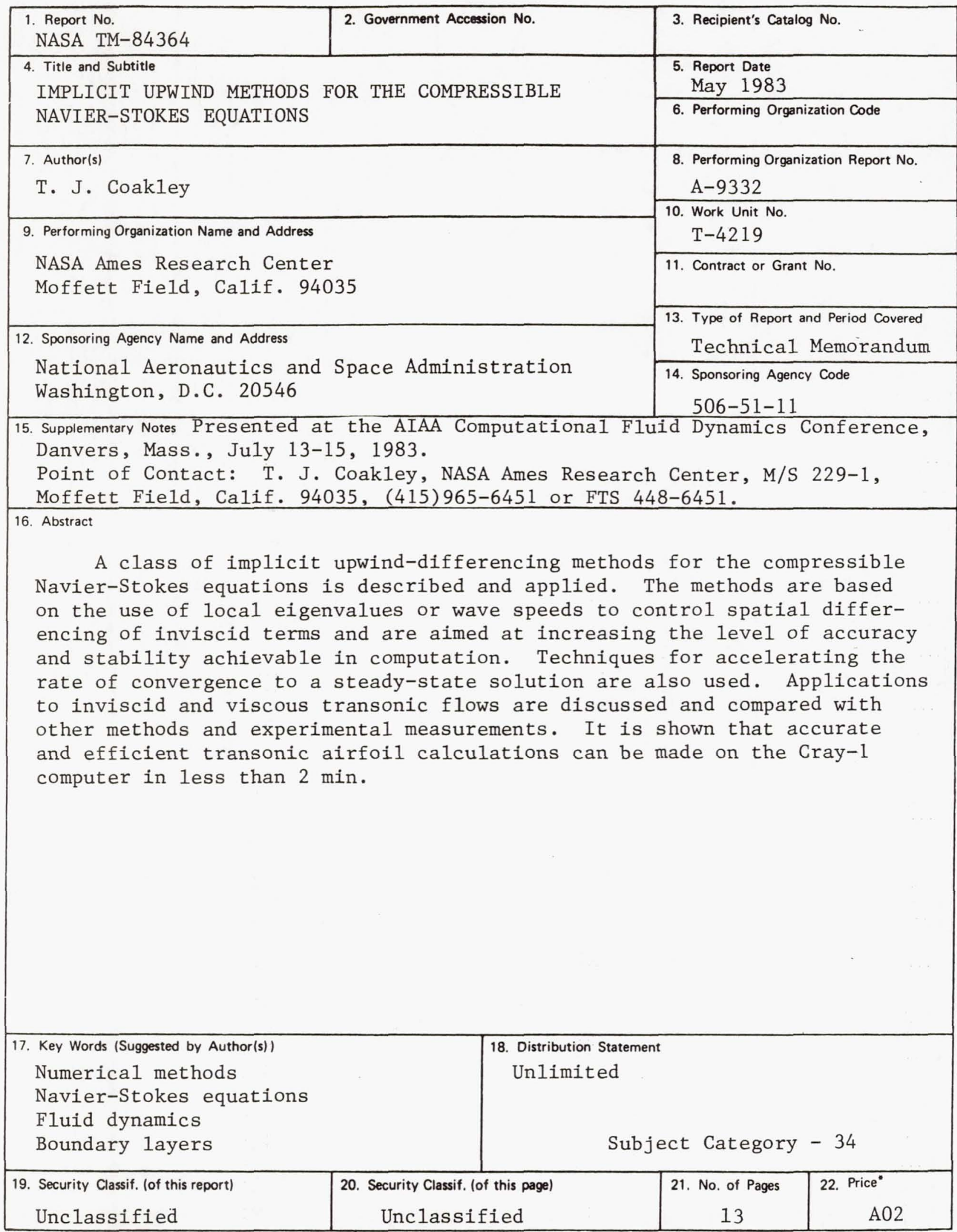

"For sale by the National Technical Information Service, Springfield, Virginia 22161 


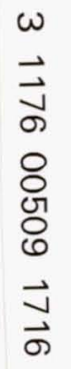


